\title{
Intrapulmonary Percussive Ventilation as an Airway Clearance Technique in Subjects With Chronic Obstructive Airway Diseases
}

\author{
Gregory Reychler PhD PT, Emilie Debier PT, Olivier Contal PhD PT, and Nicolas Audag PT
}

\begin{abstract}
BACKGROUND: Airway clearance techniques are regularly proposed as a part of the treatment in chronic obstructive airway diseases. Intrapulmonary percussive ventilation (IPV) is used as an airway clearance technique in patients affected by excessive lung secretions. The aim of this systematic review is to summarize the physiological and clinical effects related to the use of IPV as an airway clearance technique in chronic obstructive airway diseases. METHODS: This systematic review followed the PRISMA guidelines. Randomized, controlled, comparative, and cohort studies investigating IPV as an airway clearance technique were identified and reviewed from 3 databases. Two reviewers independently assessed study quality and reviewed the selected studies. RESULTS: 278 subjects from 12 studies were included in the final analysis, with 3 diseases studied. Only one of the included studies had a sample size $>50$ subjects. The main findings showed that IPV improves gas exchange during exacerbation and could reduce the hospital length of stay for patients with COPD. In subjects with cystic fibrosis, neither lung function nor other parameters were improved. CONCLUSIONS: The systematic use of IPV as an airway clearance technique in chronic obstructive airway diseases is not supported by sufficiently strong evidence to recommend routine use in this patient population. Key words: intrapulmonary percussive ventilation; airway clearance; physiotherapy; cystic fibrosis; COPD. [Respir Care 2018;63(5):620-631. (C 2018 Daedalus Enterprises]
\end{abstract}

\section{Introduction}

Airway secretions are one of the pathological components of the different chronic obstructive airway diseases.

\footnotetext{
Dr Reychler and Mr Audag are affiliated with the Institut de Recherche Expérimentale et Clinique (IREC), Pôle de Pneumologie, ORL \& Dermatologie, Université Catholique de Louvain, Brussels, Belgium. Dr Reychler and Ms Debier are affiliated with the Service de Pneumologie, Cliniques Universitaires Saint-Luc, Brussels, Belgium. Dr Contal is affiliated with the University of Applied Sciences and Arts Western Switzerland (HES-SO), Lausanne, Switzerland. Mr Audag is affiliated with the Département de Pédiatrie, Cliniques Universitaires Saint-Luc, Brussels, Belgium.
}

Dr Reychler received a grant from the Institut de Recherche Expérimentale et Clinique (Université Catholique de Louvain, Brussels, Belgium). Mr Audag received funding from Fund Eliane Lagast for his PhD scholarship.

Correspondence: Gregory Reychler PhD, PT, Pneumology Unit, Cliniques Universitaires Saint-Luc (UCL), Avenue Hippocrate 10, 1200 Brussels, Belgium. E-mail: gregory.reychler@uclouvain.be.

DOI: $10.4187 /$ respcare.05876
Therefore, airway clearance techniques are regularly used with patients who have these diseases. Airway clearance techniques aim to decrease airway resistance, improve gas exchange, and reduce respiratory load by improving airway clearance. While its efficiency is often debated, airway clearance techniques remain widely prescribed in the treatment of many chronic obstructive airway diseases..$^{1-3}$ Conventional airway clearance techniques, including postural drainage, percussion, and vibration have been described in the early literature. ${ }^{4} \mathrm{New}$ and different techniques derived from manual or instrumental interventions have appeared since then. These new techniques, however, are not always supported by well-designed, randomized studies..$^{5-7}$

Intrapulmonary percussive ventilation (IPV) derives from high-frequency percussive ventilation, which was initially applied to treat respiratory failure after smoke inhalation or burns..$^{8-11}$ It is a pressure-limited, time-cycled, high-frequency mode of ventilation that delivers sub-physiologic tidal volumes. Since its emergence, IPV has been progressively used in patients affected by excessive respiratory secretions or to treat atelectasis. ${ }^{12-18}$

In IPV, a pneumatic device (ie, a phasitron) is connected to a nebulizer. It is used to improve airway clear- 
Table 1. Inclusion Criteria

\begin{tabular}{ll}
\hline \hline \multicolumn{1}{c}{ Category } & \\
\hline $\begin{array}{l}\text { Population } \\
\text { Interventions }\end{array}$ & Subjects $>5$ y old with obstructive disease (cystic fibrosis, asthma, COPD, bronchiectasis), stable or exacerbated \\
Comparator & Use of intrapulmonary percussive ventilation as airway clearance technique \\
Outcomes & No airway clearance technique used, placebo or other airway clearance techniques \\
Period & Blood gas, lung function, sputum, symptoms, exercise capacity or tolerance, hospitalization, quality of life \\
Language & $1995-2017$ \\
Kind of studies & French or English \\
\hline
\end{tabular}

ance, to deliver medications, or to promote an adequate level of secretion hydration. This modality intermittently delivers small volumes at high frequencies, creating percussions in the lungs. ${ }^{19}$ A face mask, mouthpiece, endotracheal tube, or tracheostomy can be used as an interface. ${ }^{20}$ The pressure, frequency, and inspiratory/expiratory ratio can be determined depending on the objectives of the treatment. ${ }^{20}$ Even though IPV is frequently used as an airway clearance technique in various chronic obstructive airway diseases, there is a wide disparity in its use. ${ }^{21}$ These differences are explained by a lack of evidence regarding physiological effects, clinical effectiveness, and the settings related to specific diseases or conditions.

The aim of this systematic review is to summarize the immediate or prolonged physiological effects (eg, gas exchange, cardiorespiratory parameters, lung function, and mechanics) and clinical effects (eg, symptoms, adverse effects, and length of hospital stay) related to the use of intrapulmonary percussive ventilation as an airway clearance technique in different chronic obstructive airway diseases in stable or acute conditions.

\section{Methods}

\section{Protocol}

The Preferred Reporting Items for Systematic Reviews and Meta-Analyses (PRISMA) guidelines were consulted during the stages of design, analysis, and reporting of this systematic review. 22 According to these guidelines, the structured search, study selection, risk-of-bias assessment of individual studies, and best-evidence synthesis for relating risk-of-bias to consistency of effect sizes were included in this review. The protocol for this review has been registered in the international prospective register of systematic reviews (PROSPERO, Registration No. CRD42017068336).

\section{Search Strategy}

PubMed, PEDro, and Scopus online databases were screened for the primary search strategy from inception to
May 2017. The key terms were obstructive, COPD, cystic fibrosis, asthma*, chronic obstructive pulmonary disease, COPD, chronic obstructive airway disease, $\mathrm{COAD}$, or chronic air flow for the patient category, and intrapulmonary percussive ventilat*, IPV, or percussionnaire for the intervention category. An asterisk $\left(^{*}\right)$ indicates a wild card character.

The full search strategy for PubMed was adapted for other databases using terms and Medical Subject Headings combined with Boolean operators. A manual search of reference lists from the identified articles, citation tracking of included articles, and use of the PubMed related articles option completed the database searches to avoid missing relevant studies.

\section{Inclusion and Exclusion Criteria}

After removal of duplicates, the retrieved abstracts were reviewed critically and independently for relevance by 2 independent investigators (ED, GR). On the basis of these abstracts, research articles were included if they involved studies evaluating immediate or prolonged primary end points of physiological effects (eg, blood gas results, cardiorespiratory parameters, lung function or lung mechanics, and sputum weight) or secondary end points of clinical effects related to IPV in one of the chronic obstructive airway diseases (COPD, cystic fibrosis [CF], asthma, and bronchiectasis). They must have been written in English or French, and they must have been classified as randomized controlled studies (RCTs), cohort/case studies, or comparative studies (Table 1). Studies of children $<5$ y old, regarding IPV out of the scope of airway clearance techniques, or including subjects with a restrictive disease were excluded, as were abstracts without full text. The investigators reviewed full-text articles when the inclusion or exclusion was unclear based on the title and abstract. Any disagreement about eligibility was resolved by a consensus meeting between 3 investigators (GR, OC, NA).

\section{Data Extraction, Study Quality Appraisal, and Risk of Bias Assessment}

Two investigators (ED, GR) extracted study details and data. Extracted data included the study design, sample 
characteristics (including number of participants, age group, disease [COPD, CF, asthma, and bronchiectasis] and its severity, and inclusion/exclusion criteria of the study), protocols used (eg, device, session and treatment duration, frequency, intensity, and other settings), and outcomes. The same 2 investigators assessed the internal validity of the randomized, controlled and crossover studies using the PEDro scale and applied the quality index developed by Downs and Black for assessing methodological quality and bias for all of the reviewed studies. ${ }^{23,24}$ The Downs and Black tool, which is composed of 27 questions, was identified in a review by the Health Technology Group as one of the most appropriate tools for the evaluation of non-randomized, controlled trials in systematic reviews. ${ }^{24,25}$ Originally, the total maximum score was 32 points, but it was modified to a maximum score of $28 .{ }^{26}$ Each non-randomized controlled study was assigned a grade of excellent (24-28 points), good (19-23 points), fair (1418 points), or poor $\left(<14\right.$ points). ${ }^{26}$

\section{Summary Measures}

The investigators considered the results of the studies when the inclusion criteria were respected. Mean comparison, adverse effects, and adherence/completion rate were reported. Results were analyzed according to the diseases of the included studies.

\section{Results}

\section{Study Selection}

A total of 109 references were retrieved in the different databases and other sources (Fig. 1). After duplicates were removed, 59 articles were screened. From this analysis, 12 studies (7 RCTs ${ }^{12,14,27-31}$ ) were included in the systematic review.

\section{Characteristics of the Studies}

The characteristics of the studies are described in Table 2.

Population and Inclusion Criteria. A total of 278 subjects were included, of whom 15 did not complete the proposed protocol. Only one of the included studies had a sample size $>50$ subjects. The mean age of included subjects was $52.7 \mathrm{y}$. One publication did not mention the age of included subjects. ${ }^{32}$

COPD. Six studies recruited subjects with COPD $(n=178) .15,27,28,31-33$ Three studies were performed in ICUs during an exacerbation, ${ }^{27,31,33}$ and 2 studies were performed in stable out-patients. ${ }^{15,28}$ Two studies clearly mentioned the subjects' characteristics at the time of

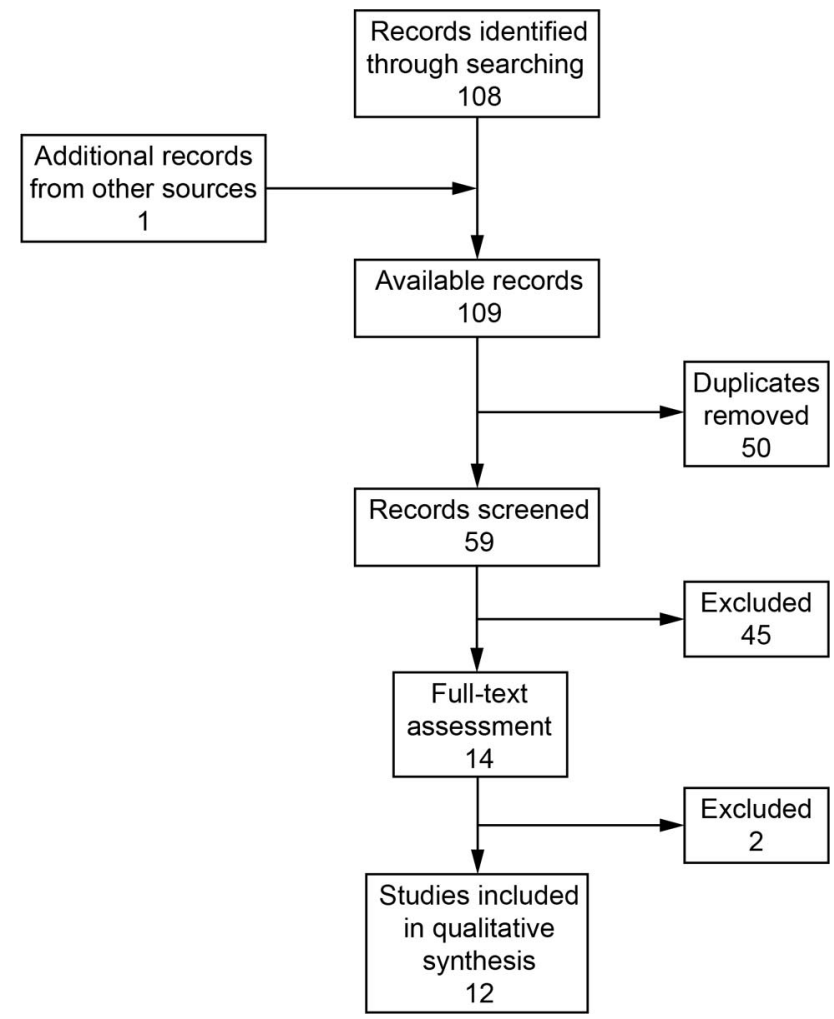

Fig. 1. Flow chart.

inclusion. ${ }^{27,31}$ Three studies excluded tracheostomized patients, ${ }^{15,31,33}$ and 4 studies required hemodynamic stability. ${ }^{15,27,31,33}$

CF and Bronchiectasis. Four studies evaluated subjects with CF $(n=78), 12,14,29,30$ and 1 study investigated subjects with bronchiectasis. ${ }^{34}$ In subjects with CF, pneumothorax and hemoptysis were considered as a contraindication in all studies. . $^{12,14,29,30}$ Only 1 study was performed during exacerbation. ${ }^{30}$ Tracheostomy was an exclusion criterion for the study involving subjects with bronchiectasis.

Interventions. IPV was mainly compared to other airway clearance techniques, including postural drainage combined with manual percussions,,$^{27,30,34}$ slow expiration with glottis opened in infralateral decubitus position (ELTGOL), ${ }^{15,27}$ autogenic drainage, ${ }^{29}$ forced expiration technique and cough, ${ }^{15}$ high-frequency chest-wall compressions (HFCWC), ${ }^{30}$ noninvasive ventilation (NIV), ${ }^{27,28}$ oscillating positive expiratory pressure (Flutter, Axcan Scandipharm, Birmingham, AL), ${ }^{14}$ or nebulization alone. ${ }^{29} \mathrm{Im}$ mediate effects were analyzed in 2 observational studies. ${ }^{32,33}$ Testa et al ${ }^{15}$ combined IPV with a pulmonary rehabilitation program.

IPV Settings. Different devices were used (IMP1, IMP2; Percussionaire, Sandpoint, ID) depending on the condi- 


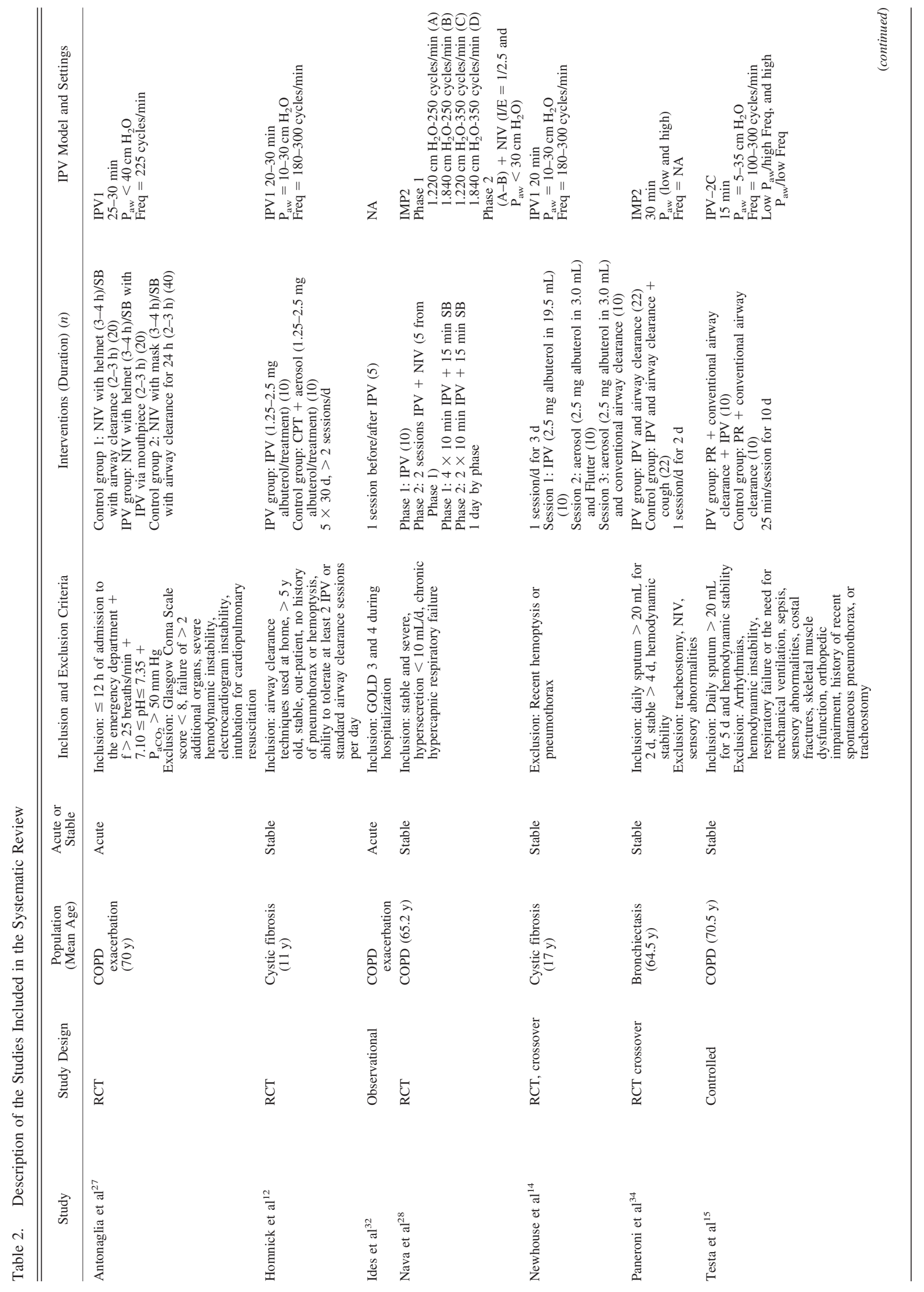




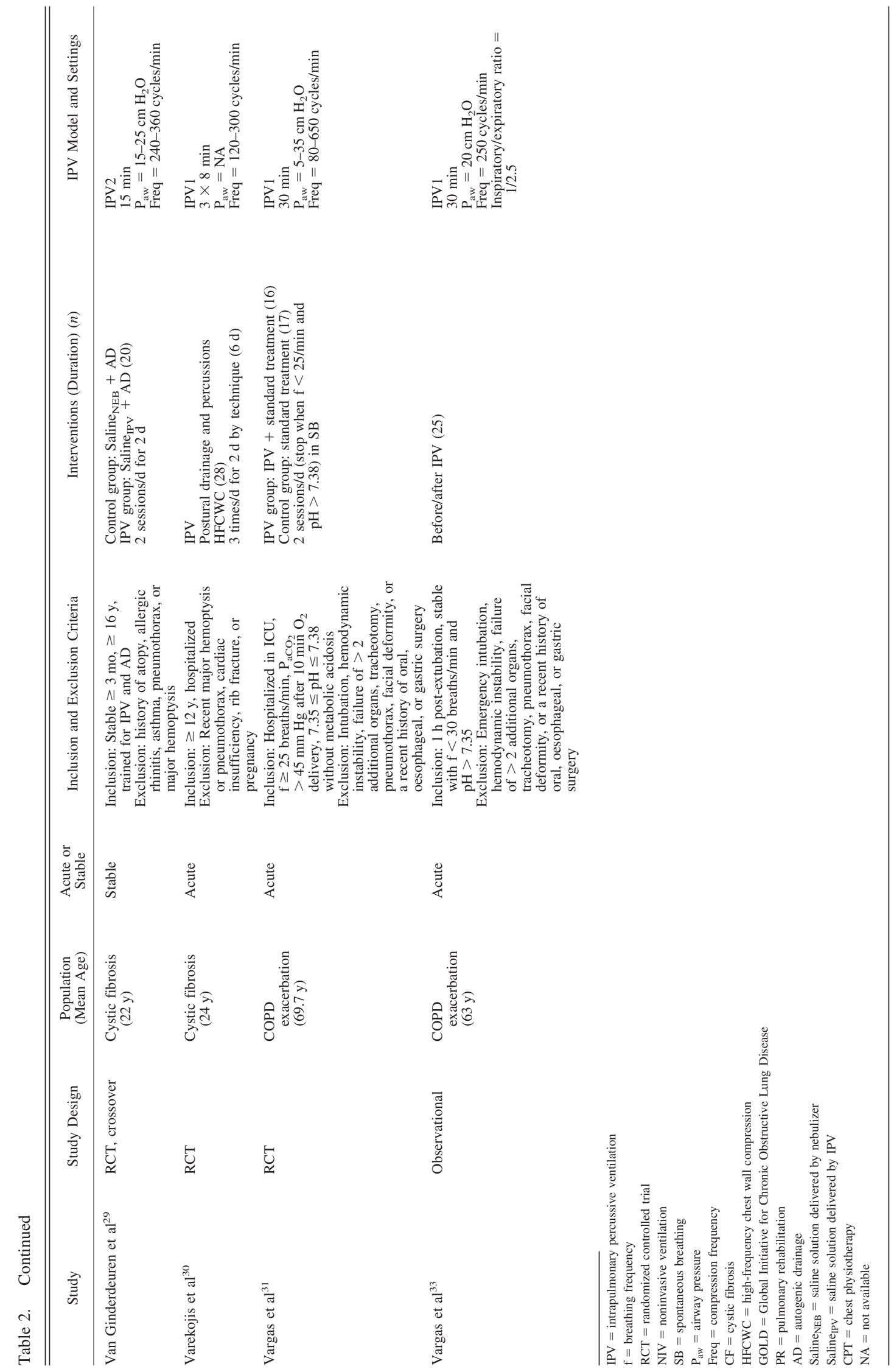


Table 3. Quality Evaluation Using the PEDro Scale for Randomized Controlled Studies

\begin{tabular}{|c|c|c|c|c|c|c|c|c|c|c|c|c|}
\hline Study & $\begin{array}{c}\text { Eligibility } \\
\text { Criteria }\end{array}$ & $\begin{array}{l}\text { Randomly } \\
\text { Allocated }\end{array}$ & $\begin{array}{l}\text { Concealed } \\
\text { Allocation }\end{array}$ & $\begin{array}{l}\text { Similar } \\
\text { Groups at } \\
\text { Baseline }\end{array}$ & $\begin{array}{l}\text { Blinding of } \\
\text { Subjects }\end{array}$ & $\begin{array}{l}\text { Blinding of } \\
\text { Therapists }\end{array}$ & $\begin{array}{l}\text { Blinding of } \\
\text { Assessors }\end{array}$ & $\begin{array}{l}\text { Data From } \\
>85 \% \text { of } \\
\text { Subjects }\end{array}$ & $\begin{array}{l}\text { Intention } \\
\text { to Treat }\end{array}$ & $\begin{array}{c}\text { Statistical } \\
\text { Comparison }\end{array}$ & $\begin{array}{l}\text { Measures of } \\
\text { Variability }\end{array}$ & $\begin{array}{l}\text { Final } \\
\text { Score }\end{array}$ \\
\hline Antonaglia et $\mathrm{al}^{27}$ & Yes & Yes & Yes & Yes & No & No & No & No & No & Yes & Yes & $5 / 10$ \\
\hline Homnick et $\mathrm{al}^{12}$ & Yes & Yes & No & Yes & No & No & No & No & No & Yes & Yes & $4 / 10$ \\
\hline Nava et $\mathrm{al}^{28}$ & No & Yes & No & Yes & No & No & No & Yes & No & Yes & Yes & $5 / 10$ \\
\hline Newhouse et $\mathrm{al}^{14}$ & Yes & Yes & No & No & No & No & Yes & No & No & No & Yes & $3 / 10$ \\
\hline Paneroni et $\mathrm{al}^{34}$ & Yes & Yes & No & No & No & No & No & Yes & No & Yes & Yes & $4 / 10$ \\
\hline Van Ginderdeuren et $\mathrm{al}^{29}$ & Yes & Yes & No & Yes & No & No & No & Yes & No & Yes & Yes & $5 / 10$ \\
\hline Varekojis et $\mathrm{al}^{30}$ & Yes & Yes & No & No & No & No & No & Yes & No & Yes & Yes & $4 / 10$ \\
\hline Vargas et $\mathrm{al}^{31}$ & Yes & Yes & Yes & Yes & No & No & No & Yes & No & Yes & Yes & $6 / 10$ \\
\hline
\end{tabular}

Table 4. Quality Evaluation Using the Downs and Black Quality Index for Randomized and Non-Randomized Controlled Studies

\begin{tabular}{|c|c|c|c|c|c|c|c|}
\hline Study & Reporting (11)* & External validity $(3)^{*}$ & Bias (7)* & Confounding $(6)^{*}$ & Power $(1)^{*}$ & Total $(28)^{*}$ & Grades $\dagger$ \\
\hline Antonaglia et $\mathrm{al}^{27}$ & 8 & 3 & 5 & 5 & 0 & 21 & Good \\
\hline Homnick et $\mathrm{al}^{12}$ & 8 & 3 & 5 & 4 & 0 & 20 & Good \\
\hline Ides et $\mathrm{al}^{32}$ & 7 & 0 & 5 & 3 & 0 & 15 & Fair \\
\hline Nava et $\mathrm{al}^{28}$ & 7 & 1 & 5 & 2 & 0 & 15 & Fair \\
\hline Newhouse et $\mathrm{al}^{14}$ & 9 & 3 & 5 & 4 & 0 & 21 & Good \\
\hline Paneroni et $\mathrm{al}^{34}$ & 8 & 3 & 5 & 4 & 0 & 20 & Good \\
\hline Testa et $\mathrm{al}^{15}$ & 9 & 1 & 6 & 3 & 0 & 19 & Fair \\
\hline Van Ginderdeuren et $\mathrm{al}^{29}$ & 8 & 3 & 5 & 4 & 0 & 20 & Good \\
\hline Varekojis et $\mathrm{al}^{30}$ & 8 & 3 & 5 & 4 & 0 & 20 & Good \\
\hline Vargas et $\mathrm{al}^{31}$ & 7 & 3 & 5 & 3 & 0 & 18 & Fair \\
\hline Vargas et $\mathrm{al}^{33}$ & 9 & 3 & 5 & 4 & 0 & 22 & Good \\
\hline
\end{tabular}

* Maximum score for each item with the Downs and Black Quality Index.

$\dagger$ Grading total score: Excellent (24-28 points); Good (19-23 points); Fair (14-18 points); Poor ( $<14$ points).

tions. Durations of the sessions were heterogeneous and varied from 15 to $40 \mathrm{~min}$; 1 study did not mention the duration of the session. ${ }^{32}$ IPV use was divided in the sessions in 2 studies. 28,30

A low frequency was set in all studies, but 6 of the 12 studies combined high and low frequency. $12,14,15,28,29,31$ With regard to the pressure settings, the authors fixed a pressure $<40 \mathrm{~cm} \mathrm{H}_{2} \mathrm{O}$ when mentioned. However, 3 studies did not mention this setting. ${ }^{30,32,34}$ Only 2 studies stated the inspiratory-expiratory ratio (1/2.5)..$^{28,31}$ A mouthpiece was used in 7 studies, ${ }^{12,14,27,29,30,33,35}$ and a face mask was used in 2 studies ${ }^{28,31} ; 3$ studies did not describe the interface. ${ }^{15,32,34}$

All studies including subjects with COPD or CF nebulized a saline solution simultaneously during the session. Two studies administered a drug (bronchodilator) during the session in subjects with CF. ${ }^{12,14}$ The bronchodilator was diluted in a large volume in 1 study. ${ }^{14}$ The study including subjects with bronchiectasis did not mention the use of a nebulized solution. ${ }^{34}$

\section{Quality and Design of the Studies}

After quality assessment, the RCTs ranged from 3 to 6 on the PEDro scale (Table 3). The median score was 5.
One RCT did not specify the eligibility criteria in the Methods section. ${ }^{28}$ The Downs and Black index ranged from 13 to 22 with a median score of 19.5 for all the included studies. Most of them were classified as "good" in the quality appraisal (Table 4). ${ }^{15,32,33}$

\section{Results of the Studies}

All the results are reviewed in Tables 5 and 6 .

Gas Exchange in COPD, CF, and Bronchiectasis. Acidbase balance was improved by IPV combined with NIV, standard treatment, or used alone during an exacerbation. ${ }^{27,31,33}$ After 1 session, $\mathrm{P}_{\mathrm{aO}_{2}}$ and $\mathrm{P}_{\mathrm{aCO}_{2}}$ improved during COPD exacerbation, ${ }^{27,31,33}$ but IPV was no more beneficial in stable condition than other airway clearance techniques when subjects were stable. ${ }^{15}$ After IPV combined with NIV, gas exchange was improved compared to CPT with NIV. ${ }^{27}$ Short- and long-term effects were observed on impaired gas exchange in subjects with COPD. This benefit was only found in acute conditions. Gas exchange was not used as an outcome measure in $\mathrm{CF}$ or in bronchiectasis. 


\section{IPV and Chronic Obstructive Airway Diseases}

Table 5. Results of the Studies in Patients With COPD

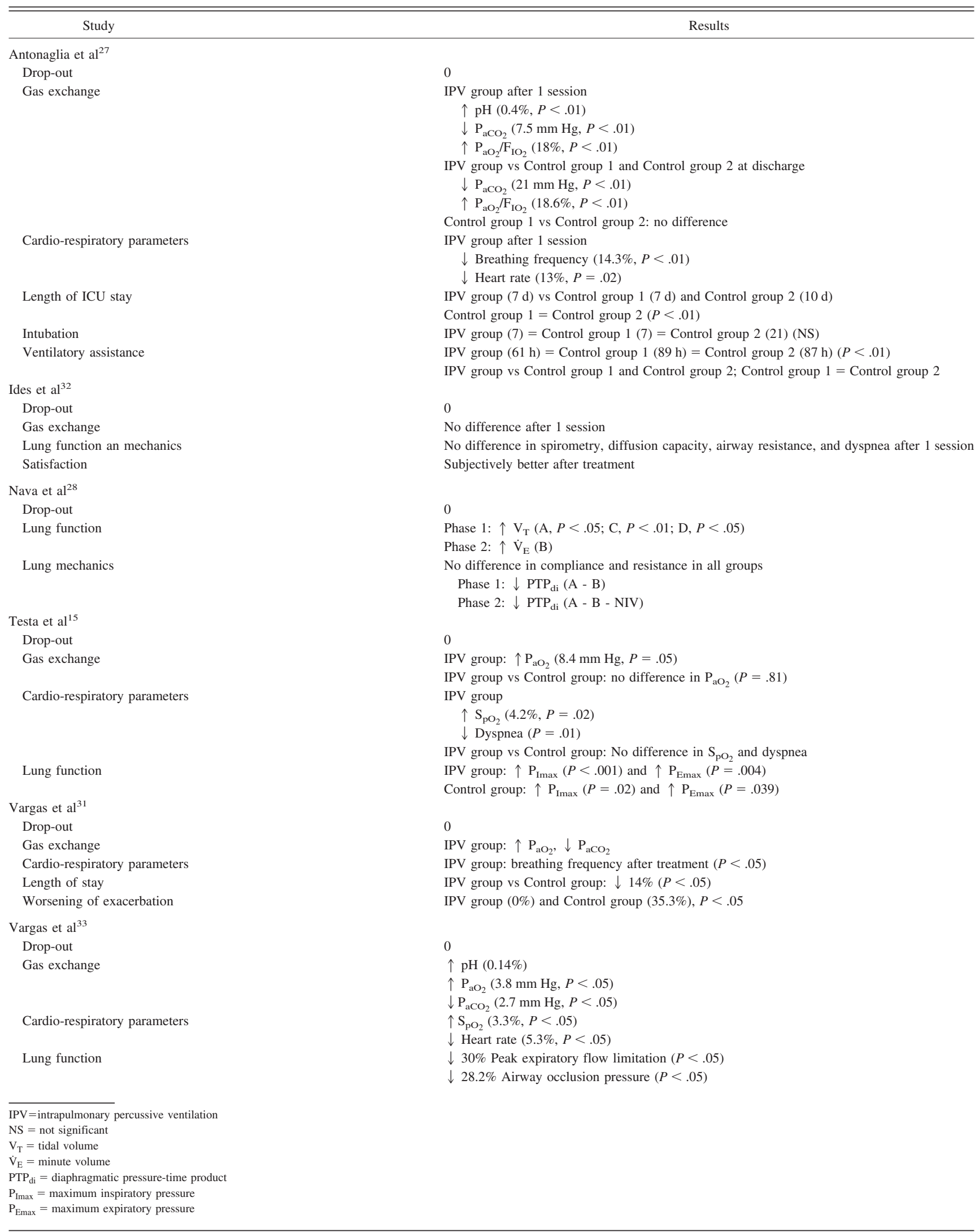




\section{IPV and Chronic Obstructive Airway Diseases}

Table 6. Results of the Studies in Patients With CF and Bronchiectasis

\begin{tabular}{|c|c|}
\hline Study & Results \\
\hline \multicolumn{2}{|l|}{ Homnick et $\mathrm{al}^{12}$} \\
\hline Drop-out & 4 (IPV group: 2, Control group: 2) \\
\hline Lung function & 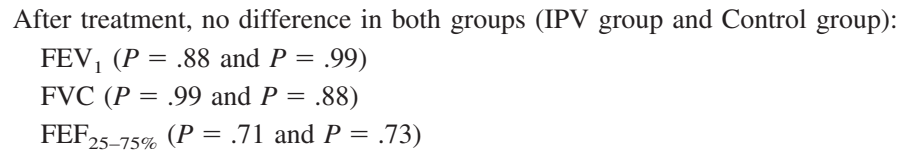 \\
\hline Length of stay & No difference \\
\hline Oral or IV antibiotic use & No difference between groups \\
\hline Satisfaction (IPV vs other airway clearance technique) & Efficacy $(75 \%)$, time-consuming $(0 \%)$, independence $(87.5 \%)$, comfort $(62.5 \%)$ \\
\hline Complications & Hemoptysis ( $n=1$ in IPV group) \\
\hline \multicolumn{2}{|l|}{ Newhouse et a $1^{14}$} \\
\hline Drop-out & 2 (infection) \\
\hline Lung function (IPV, Flutter, or airway clearance technique) & No change in static lung volume after $1 \mathrm{~h}$ and $4 \mathrm{~h}$ \\
\hline IPV & $\uparrow \mathrm{FEV}_{1}$ after $1 \mathrm{~h}(P=.02)$ \\
\hline Flutter & $\uparrow \mathrm{FVC}$ after $1 \mathrm{~h}(P=.02)$ \\
\hline & $\uparrow \mathrm{FEV}_{1}$ after $1 \mathrm{~h}(P=.033)$ and $4 \mathrm{~h}(P=.048)$ \\
\hline Sputum & No change in sputum wet weight after $1 \mathrm{~h}$ and $4 \mathrm{~h}$ \\
\hline Adverse effects & No \\
\hline \multicolumn{2}{|l|}{ Paneroni et $\mathrm{al}^{34}$} \\
\hline Drop-out & 0 \\
\hline Cardio-respiratory parameters & $\begin{array}{l}\text { IPV group } \\
\quad \downarrow \text { breathing frequency }(P=.02) \\
\quad \downarrow \text { dyspnea }(P=.04) \\
\quad \downarrow \text { heart rate }(P=.002) \\
\quad \mathrm{S}_{\mathrm{pO}_{2}}(\mathrm{NS}) \\
\text { IPV group vs Control group: only breathing frequency was different }(P=.047)\end{array}$ \\
\hline Sputum & $\begin{array}{l}\text { IPV group: no difference in volume and wet/dry weight } \\
\text { IPV group vs Control group: no difference }\end{array}$ \\
\hline Satisfaction & $\begin{array}{l}\text { IPV group: } \downarrow \text { sensation of encumbrance and } \downarrow \text { discomfort }(P=.03) \\
\text { IPV group vs Control group: no difference }\end{array}$ \\
\hline Adverse effects & Both groups: $27 \%$ (dry throat, nausea, and/or fatigue) \\
\hline \multicolumn{2}{|l|}{ Van Ginderdeuren et $\mathrm{al}^{29}$} \\
\hline Drop-out & No data \\
\hline Cardio-respiratory parameters & IPV group vs Contol group: no difference in $\mathrm{S}_{\mathrm{pO}_{2}}$, heart rate, and dyspnea \\
\hline Sputum & $\begin{array}{l}\text { IPV group vs Control group: no difference in sputum wet weight } \\
\text { No difference in sputum wet weight } \\
\text { Autogenic drainage increased sputum wet weight in both groups }(P<.001)\end{array}$ \\
\hline \multicolumn{2}{|l|}{ Varekojis et $\mathrm{al}^{30}$} \\
\hline Drop-out & 4 \\
\hline Sputum & $\begin{array}{l}\text { Wet weight: IPV }>\text { HFCWC }(P=.035) \\
\text { No difference in dry weight }(P=.17)\end{array}$ \\
\hline Satisfaction & $\begin{array}{l}\text { No difference between techniques in comfort, convenience, efficacy, ease of } \\
\quad \text { use, and global satisfaction } \\
\text { Preferred technique: } \\
10 \text { subjects preferred HFCWC } \\
7 \text { subjects preferred IPV } \\
7 \text { subjects preferred postural drainage and percussion }\end{array}$ \\
\hline $\begin{array}{l}\mathrm{CF}=\text { cystic fibrosis } \\
\mathrm{IPV}=\text { intrapulmonary percussive ventilation } \\
\mathrm{FEF}_{25-75 \%}=\text { forced expiratory flow between } 25-75 \% \text { of vital capacity } \\
\text { NS = not significant } \\
\text { HFCWC = high-frequency chest wall compression }\end{array}$ & \\
\hline
\end{tabular}


Cardiorespiratory Parameters, Lung Function, and Lung Mechanics in COPD, CF, and Bronchiectasis. In subjects with COPD in stable conditions and during exacerbation, all cardio-respiratory parameters, lung function, and lung mechanics decreased with IPV. ${ }^{27,31,33}$ Compared to spontaneous breathing, the tidal volume increase associated with IPV was related to the settings $\left(1.220 \mathrm{~cm} \mathrm{H}_{2} \mathrm{O}-250\right.$ c/min, $1.220 \mathrm{~cm} \mathrm{H}_{2} \mathrm{O}-350 \mathrm{c} / \mathrm{min}$, and $1.840 \mathrm{~cm} \mathrm{H}_{2} \mathrm{O}-350$ $\mathrm{c} / \mathrm{min}$ ), but a reduced diaphragmatic loading was only observed for 1 setting $\left(1.220 \mathrm{~cm} \mathrm{H}_{2} \mathrm{O}-250 \mathrm{c} / \mathrm{min}\right){ }^{28}$ After $1 \mathrm{~d}$ of treatment in stable subjects, 1 study showed an improvement in inspiratory and expiratory muscle strength. ${ }^{15}$

The cardiorespiratory parameters were not modified by IPV in subjects with CF. ${ }^{29}$ Only dyspnea and respiratory frequency improved after 1 session of IPV in subjects with bronchiectasis. ${ }^{34}$ No change was observed in static or dynamic lung volume in subjects with CF. ${ }^{12,14}$ Based on these results, the benefits related to IPV in these disease states do not appear to be evident.

Sputum Weight in COPD, CF, and Bronchiectasis. Sputum was not considered as an outcome in studies evaluating the effects of IPV in subjects with COPD. All studies related to $\mathrm{CF}$ were short-term studies. Sputum was collected in 3 studies of subjects with $\mathrm{CF}^{14,29,30}$ and in 1 study of subjects with bronchiectasis. ${ }^{34}$ All of these studies compared IPV with other airway clearance techniques (eg, autogenic drainage, HFCWC). Increased sputum wet weight was observed with IPV compared to HFCWC in subjects hospitalized for an exacerbation and after autogenic drainage combined with IPV compared to IPV alone in stable subjects. ${ }^{29,30}$ These short-term studies administered saline solution by nebulization simultaneously to the IPV session. However, when investigated, no difference was observed in sputum dry weight. ${ }^{30}$ In subjects with productive bronchiectasis, the immediate efficacy of IPV and other airway clearance techniques was not different. ${ }^{34}$ These results are not sufficient to make conclusions about the efficacy of IPV on sputum in patients with CF or bronchiectasis.

\section{Length of Hospital Stay and Other Clinical Outcomes} in COPD, CF, and Bronchiectasis. The length of hospital stay was reduced by IPV compared to other airway clearance techniques or to a classical medical treatment alone in 2 studies in subjects with COPD during exacerbation. ${ }^{27,31}$ In 1 study, a decrease in the need for mechanical ventilation was observed. ${ }^{27}$ Only 1 long-term study focused on these outcomes by comparing IPV and conventional airway clearance techniques in subjects with $\mathrm{CF}$. Neither the duration of antibiotics use nor the length of hospital stay were modified by IPV in these subjects. ${ }^{12}$
Satisfaction. In 1 long-term study, $>75 \%$ of subjects with CF demonstrated higher satisfaction regarding efficacy, independency, and comfort with IPV than with other airway clearance techniques. This was not related to the time required for the treatment. ${ }^{12}$ This benefit was not found in another study in which IPV was compared with HFCWC or postural drainage with percussion. ${ }^{30}$

\section{Adverse Effects and Drop-Outs in COPD, CF, and Bron-} chiectasis. Few adverse effects related to IPV were mentioned in the different studies. In subjects with COPD, 2 studies revealed complications or discomfort. Even though some subjects were intubated after inclusion in 1 study, it was not related to IPV. ${ }^{27}$ In another study, 2 subjects did not tolerate settings with a higher frequency of percussions $\left(1.220 \mathrm{~cm} \mathrm{H}_{2} \mathrm{O}-350 \mathrm{c} / \mathrm{min}\right.$ and $1.840 \mathrm{~cm}$ $\left.\mathrm{H}_{2} \mathrm{O}-350 \mathrm{c} / \mathrm{min}\right){ }^{28}$

Only 1 study observed mild hemoptysis associated with a Pseudomonas aeruginosa infection in 1 subject with CF.12 Drop-outs were noted in 3 studies in subjects with CF. ${ }^{12,14,30}$ Two subjects stopped the treatment for pulmonary infection, ${ }^{14}$ and 8 others without detailed reasons; ${ }^{12,30}$ the number of drop-outs were not different in the IPV group. Minor adverse effects were also found in $27 \%$ of subjects with bronchiectasis. ${ }^{34}$

\section{Discussion}

This systematic review highlights that IPV provides insufficient and heterogeneous results, which precludes IPV from being routinely recommended for different chronic obstructive airway diseases. In subjects with stable COPD, there is a lack of evidence regarding the use of IPV. However, during exacerbation, IPV may improve gas exchange and reduce hospital length of stay. In patients with CF or bronchiectasis, the efficacy of IPV has not been demonstrated.

Studies evaluating IPV as an airway clearance technique investigated mainly 2 diseases at the time of the systematic review (CF and COPD). Only 1 study focused on bronchiectasis. ${ }^{34}$ Compared to the total number of publications regarding airway clearance in these specific diseases, IPV is poorly studied. Combined with poor statistical power, the internal and external validity of the reviewed studies explain why IPV lacks evidence as an effective airway clearance technique in chronic obstructive airway disease, and it is difficult to draw robust conclusions.

We observed broad heterogeneity in the protocols of the studies related to the use of IPV in chronic obstructive airway diseases. The studies considered 6 different airway clearance techniques as comparators, and 3 of the studies used techniques that are not recognized as airway clearance techniques (NIV and nebulization). ${ }^{27,28,36}$ With regard to the inclusion and exclusion criteria of the studies, 
few adverse events were noted in the reviewed studies. The risk of hemoptysis is the main adverse effect to be considered, and this was observed only in subjects with CF.

The settings were heterogeneous even though all of the studies used at least 1 setting based on a low frequency, which is consistent with findings related to the specific settings to promote the clearance of lung secretions. ${ }^{37}$ Indeed, it is well demonstrated that adapting the settings for IPV modifies the resulting effects on the lungs. Surprisingly, while the inspiratory/expiratory time ratio seems to be an essential setting to promote mobilization of sputum, this parameter was rarely mentioned in the protocols. ${ }^{20}$

Contrary to subjects with $\mathrm{CF}$, subjects with COPD were mainly investigated during exacerbation in the retrieved studies. This is explained by the difference in the respective objectives of the IPV in both diseases. In patients with $\mathrm{CF}$, the main objective is to clear secretions, whereas in patients with COPD, IPV targets an improvement of gas exchange and the parameters related to the exacerbations. IPV is probably more effective as a ventilator support method than an airway clearance technique in COPD.

IPV demonstrated immediate and prolonged effects on peripheral oxygen saturation and gas exchange both in stable subjects with COPD and during exacerbations. ${ }^{15,27,31,33}$ These effects highlight possible lung recruitment obtained by this technique. A similar effect was found in subjects with atelectasis ${ }^{17,18}$ and obesit. ${ }^{38}$ This could be partly due to the positive pressure generated by IPV and to the improvement in lung ventilation. We could hypothesize that improved airway clearance also contributes to this result. However, even though other studies showed a greater expectorated sputum amount with different airway clearance techniques in COPD, this was not verified in this systematic review when focusing exclusively on IPV. 3,39

During exacerbation, a promising benefit was observed in 2 studies on the length of hospital stay. ${ }^{27,31}$ Such an effect was rarely found in studies related to airway clearance techniques, and it would be useful to further investigate this benefit. In the postextubation phase, 1 study demonstrated an immediate reduction of expiratory flow limitation in subjects with COPD, which may result in reduced dynamic hyperinflation. ${ }^{33}$ In stable subjects with COPD, the main immediate effect observed with IPV was a decrease in diaphragmatic work, which could be related to reduced expiratory flow limitation when positive pressure is applied or due to possible bypassing of intrinsic positive end-expiratory pressure. ${ }^{28}$ Based on these results, a trial of IPV may be warranted in carefully selected stable patients with COPD.

Studies in CF focused mainly on lung function and sputum, and all but one were performed in stable subjects. An immediate effect on lung function was observed after 1 session, ${ }^{14}$ but this improvement was not maintained long term. ${ }^{12}$ This immediate effect was surprising. The principal aim of airway clearance techniques is to improve the elimination of sputum. Thus, we could hypothesize that reducing sputum in the lungs would improve vital capacity. However, even though a recent study in bronchiectasis observed such an effect, ${ }^{40}$ it was rarely found with other airway clearance techniques in subjects with CF. ${ }^{41-43}$ In this systematic review, we did not find any benefit of IPV on airway clearance, contrary to what was demonstrated in subjects with Duchenne muscular dystrophy. ${ }^{19}$ The wet weight of sputum increased with IPV compared to other airway clearance techniques in 1 study including 3 daily sessions, ${ }^{30}$ but the combination of IPV and autogenic drainage was as efficient on sputum as autogenic drainage used alone. ${ }^{29}$ The immediate benefit observed on wet weight was probably explained by normal saline delivered simultaneously with IPV because the dry weight of sputum was not different in the same study. Moreover, cold and dry air delivered to the lungs by the phasitron may cause mucus on the respiratory epithelium to become more viscous due insufficient moisture carried by cold air. Indeed, the isothermic saturation boundary moves distally when cold and dry gases are inhaled because a greater proportion of the airways have to participate in heat and moisture exchange.

During IPV, a greater amount of water evaporates from the mucus to improve the humidification of the dry and cold gas. ${ }^{44}$ This is particularly important when the nose is bypassed, and this effect likely affects airway clearance negatively. The nebulizer provided with the IPV device is insufficient to promote adequate humidification, so a heated humidifier on the inspiratory line downstream is required. ${ }^{45}$ We can also assume that the amount of secretions in the lung at the time of the session influences the airway clearance effect related to IPV, as Toussaint et al ${ }^{19}$ clearly demonstrated in neuromuscular subjects. The considerable inter-subject variability in the recovered sputum as illustrated by coefficients of variation $>100 \%$ in the sputum wet weight reinforces this hypothesis. It is not surprising that inspiratory and expiratory muscle strength does not seem to be improved by the adjunction of IPV during a pulmonary rehabilitation program. ${ }^{15} \mathrm{We}$ postulated that it could be more interesting to analyze other outcomes such as gas exchange in subjects with severe CF. Moreover, the effect of IPV on early obstructive lung disease, early disease progression, and ventilation heterogeneity could be investigated in terms of lung clearance index in patients with CF. ${ }^{46,47}$ Indeed, the benefit of IPV in patients with CF could be related to the positive airway pressure generated by the device, similarly to the ventilator support offered by IPV in patients with COPD.

In this systematic review, 2 studies delivered bronchodilators by nebulization with IPV. ${ }^{12,14}$ This was surprising due to the evidence of poor efficiency of this delivery method in spontaneously breathing subjects. ${ }^{48,49}$ However, 
the concerned studies were performed previous to these findings.

The main limitation of this systematic review was the broad heterogeneity of the results and settings in the reviewed studies. This is a common limitation regarding airway clearance techniques. The small number of subjects included in this systematic review limits the external validity of these studies. Moreover, the reviewed studies were performed in subjects experiencing acute or chronic conditions, and investigated immediate or prolonged effects. The lack of evidence for IPV is due largely to the absence of standardized protocols and the difficulty of finding an outcome to evaluate the efficacy of the technique. Moreover, many studies are underpowered as illustrated by low Downs and Black scores. Further studies on large and homogeneous samples of subjects, with standardized protocols (fixed settings) in the different disease states, are needed. The way to use IPV in each specific condition should be established, depending on the physiological effects. Indeed, Fornasa et al demonstrated that the chosen frequency influences the pressure and the flow generated by IPV. ${ }^{35}$ Similar studies should be performed to better understand the impact of the different settings.

\section{Conclusions}

In conclusion, the systematic use of IPV in different chronic obstructive airway diseases as an airway clearance technique is not supported by sufficiently strong evidence to recommend its routine use. However, IPV could offer some benefits in patients with COPD during exacerbation by improving gas exchange and by possibly reducing the length of hospital stay. IPV is probably more a ventilatory support method than an airway clearance technique in these patients. In $\mathrm{CF}$, even if the subjects mentioned a higher satisfaction regarding efficacy, independency, and comfort with IPV, its routine use cannot be supported. Further studies are required, particularly in patients with $\mathrm{CF}$, to evaluate IPV as a tool for airway clearance but also for improving early obstructive lung disease and lung ventilation heterogeneity in patients with $\mathrm{CF}$ or bronchiectasis. Moreover, patients with COPD in a stable condition should be better investigated.

\section{REFERENCES}

1. Health Quality Ontario. Airway clearance devices for cystic fibrosis: an evidence-based analysis. Ont Health Technol Assess Ser 2009; 9(26): $1-50$.

2. Marks JH. Airway clearance devices in cystic fibrosis. Paediatr Respir Rev 2007;8(1):17-23.

3. Hill K, Patman S, Brooks D. Effect of airway clearance techniques in patients experiencing an acute exacerbation of chronic obstructive pulmonary disease: a systematic review. Chron Respir Dis 2010; 7(1):9-17.
4. Nelson HP. Postural drainage of the lungs. Br Med J 1934;2(3840): 251-255.

5. Figuls M, Gine-Garriga M, Granados RC, Perrotta C, Vilaro J. Chest physiotherapy for acute bronchiolitis in paediatric patients between 0 and 24 months old. Cochrane Database Syst Rev 2016;2:CD004873.

6. Morrison L, Innes S. Oscillating devices for airway clearance in people with cystic fibrosis. Cochrane Database Syst Rev 2017;5: CD006842.

7. Yang M, Yuping Y, Yin X, Wang BY, Wu T, Liu GJ, et al. Chest physiotherapy for pneumonia in adults. Cochrane Database Syst Rev 2010;(2):CD006338.

8. Fitzpatrick JC, Cioffi WG, Jr. Ventilatory support following burns and smoke-inhalation injury. Respir Care Clin N Am 1997;3(1):2149.

9. Hall JJ, Hunt JL, Arnoldo BD, Purdue GF. Use of high-frequency percussive ventilation in inhalation injuries. J Burn Care Res 2007; 28(3):396-400

10. Hiller KN, Morgan CK. High-frequency percussive ventilation for severe inhalation injury. Anesthesiology 2014;120(4):998.

11. Reper P, Van BR, Van LK, Van LP, Vanderkelen A. High-frequency percussive ventilation in burn patients: hemodynamics and gas exchange. Burns 2003;29(6):603-608.

12. Homnick DN, White F, de CC. Comparison of effects of an intrapulmonary percussive ventilator to standard aerosol and chest physiotherapy in treatment of cystic fibrosis. Pediatr Pulmonol 1995;20(1): 50-55.

13. Natale JE, Pfeifle J, Homnick DN. Comparison of intrapulmonary percussive ventilation and chest physiotherapy. A pilot study in patients with cystic fibrosis. Chest 1994;105(6):1789-1793.

14. Newhouse PA, White F, Marks JH, Homnick DN. The intrapulmonary percussive ventilator and flutter device compared to standard chest physiotherapy in patients with cystic fibrosis. Clin Pediatr (Phila) 1998;37(7):427-432.

15. Testa A, Galeri S, Villafane JH, Corbellini C, Pillastrini P, Negrini S. Efficacy of short-term intrapulmonary percussive ventilation in patients with chronic obstructive pulmonary disease. Disabil Rehabil 2015;37(10):899-903.

16. Birnkrant DJ, Pope JF, Lewarski J, Stegmaier J, Besunder JB. Persistent pulmonary consolidation treated with intrapulmonary percussive ventilation: a preliminary report. Pediatr Pulmonol 1996;21(4): 246-249.

17. Deakins K, Chatburn RL. A comparison of intrapulmonary percussive ventilation and conventional chest physiotherapy for the treatment of atelectasis in the pediatric patient. Respir Care 2002;47(10): 1162-1167.

18. Yen Ha TK, Bui TD, Tran AT, Badin P, Toussaint M, Nguyen AT. Atelectatic children treated with intrapulmonary percussive ventilation via a face mask: clinical trial and literature overview. Pediatr Int 2007;49(4):502-507.

19. Toussaint M, De WH, Steens M, Soudon P. Effect of intrapulmonary percussive ventilation on mucus clearance in duchenne muscular dystrophy patients: a preliminary report. Respir Care 2003;48(10): 940-947.

20. Toussaint M, Guillet MC, Paternotte S, Soudon P, Haan J. Intrapulmonary effects of setting parameters in portable intrapulmonary percussive ventilation devices. Respir Care 2012;57(5):735-742.

21. Bott J, Blumenthal S, Buxton M, Ellum S, Falconer C, Garrod R, et al. Guidelines for the physiotherapy management of the adult, medical, spontaneously breathing patient. Thorax 2009;64(Suppl 1):151.

22. Moher D, Shamseer L, Clarke M, Ghersi D, Liberati A, Petticrew M, et al. Preferred reporting items for systematic review and metaanalysis protocols (PRISMA-P) 2015 statement. Syst Rev 2015;4:1. 


\section{IPV and Chronic Obstructive Airway Diseases}

23. de Morton NA. The PEDro scale is a valid measure of the methodological quality of clinical trials: a demographic study. Aust J Physiother 2009;55(2):129-133.

24. Downs SH, Black N. The feasibility of creating a checklist for the assessment of the methodological quality both of randomised and non-randomised studies of health care interventions. J Epidemiol Community Health 1998;52(6):377-384.

25. Teasell R, Bayona N, Marshall S, Cullen N, Bayley M, Chundamala $\mathrm{J}$, et al. A systematic review of the rehabilitation of moderate to severe acquired brain injuries. Brain Inj 2007;21(2):107-112.

26. O'Connor SR, Tully MA, Ryan B, Bradley JM, Baxter GD, McDonough SM. Failure of a numerical quality assessment scale to identify potential risk of bias in a systematic review: a comparison study. BMC Res Notes 2015;8:224.

27. Antonaglia V, Lucangelo U, Zin WA, Peratoner A, De SL, Capitanio $\mathrm{G}$, et al. Intrapulmonary percussive ventilation improves the outcome of patients with acute exacerbation of chronic obstructive pulmonary disease using a helmet. Crit Care Med 2006;34(12):29402945.

28. Nava S, Barbarito N, Piaggi G, De ME, Cirio S. Physiological response to intrapulmonary percussive ventilation in stable COPD patients. Respir Med 2006;100(9):1526-1533.

29. Van Ginderdeuren F, Verbanck S, Van CK, Vanlaethem S, Schuermans D, Vincken W, et al. Chest physiotherapy in cystic fibrosis: short-term effects of autogenic drainage preceded by wet inhalation of saline versus autogenic drainage preceded by intrapulmonary percussive ventilation with saline. Respiration 2008;76(2):175-180.

30. Varekojis SM, Douce FH, Flucke RL, Filbrun DA, Tice JS, McCoy $\mathrm{KS}$, et al. A comparison of the therapeutic effectiveness of and preference for postural drainage and percussion, intrapulmonary percussive ventilation, and high-frequency chest wall compression in hospitalized cystic fibrosis patients. Respir Care 2003;48(1):24-28.

31. Vargas F, Bui HN, Boyer A, Salmi LR, Gbikpi-Benissan G, Guenard $\mathrm{H}$, et al. Intrapulmonary percussive ventilation in acute exacerbations of COPD patients with mild respiratory acidosis: a randomized controlled trial [ISRCTN17802078]. Crit Care 2005;9(4):R382-R389.

32. Ides K, Vos W, De BL, Vissers D, Claes R, Leemans G, et al. Acute effects of intrapulmonary percussive ventilation in COPD patients assessed by using conventional outcome parameters and a novel computational fluid dynamics technique. Int J Chron Obstruct Pulmon Dis 2012;7:667-671.

33. Vargas F, Boyer A, Bui HN, Guenard H, Gruson D, Hilbert G. Effect of intrapulmonary percussive ventilation on expiratory flow limitation in chronic obstructive pulmonary disease patients. J Crit Care 2009;24(2):212-219.

34. Paneroni M, Clini E, Simonelli C, Bianchi L, Degli AF, Vitacca M. Safety and efficacy of short-term intrapulmonary percussive ventilation in patients with bronchiectasis. Respir Care 2011;56(7):984-988.

35. Fornasa E, Ajcevic M, Accardo A. Characterization of the mechanical behavior of intrapulmonary percussive ventilation. Physiol Meas 2013;34(12):1583-1592.
36. Van Ginderdeuren F, Vandenplas Y, Deneyer M, Vanlaethem S, Buyl R, Kerckhofs E. Effectiveness of airway clearance techniques in children hospitalized with acute bronchiolitis. Pediatr Pulmonol 2017;52(2):225-231.

37. Riffard G, Toussaint M. [Intrapulmonary percussion ventilation: operation and settings]. Rev Mal Respir 2012;29(2):347-354.

38. Tsuruta R, Kasaoka S, Okabayashi K, Maekawa T. Efficacy and safety of intrapulmonary percussive ventilation superimposed on conventional ventilation in obese patients with compression atelectasis. J Crit Care 2006;21(4):328-332.

39. Bellone A, Spagnolatti L, Massobrio M, Bellei E, Vinciguerra R, Barbieri A, et al. Short-term effects of expiration under positive pressure in patients with acute exacerbation of chronic obstructive pulmonary disease and mild acidosis requiring non-invasive positive pressure ventilation. Intensive Care Med 2002;28(5):581-585.

40. Poncin W, Reychler G, Leeuwerck N, Bauwens N, Aubriot AS, Nader C, et al. Short-term effect of autogenic drainage on ventilation inhomogeneity in adult subjects with stable non-cystic fibrosis bronchiectasis. Respir Care 2017;62(5):524-531.

41. Elkins MR, Jones A, van der Schans C. Positive expiratory pressure physiotherapy for airway clearance in people with cystic fibrosis. Cochrane Database Syst Rev 2006;(2):CD003147.

42. Morrison L, Agnew J. Oscillating devices for airway clearance in people with cystic fibrosis. Cochrane Database Syst Rev 2009;(1): CD006842.

43. Warnock L, Gates A. Chest physiotherapy compared to no chest physiotherapy for cystic fibrosis. Cochrane Database Syst Rev 2015; (12):CD001401.

44. McNulty G, Eyre L. Humidification in anaesthesia and critical care. BJA Education 2015;15(3):131-135.

45. Dellamonica J, Louis B, Lyazidi A, Vargas F, Brochard L. Intrapulmonary percussive ventilation superimposed on conventional ventilation: bench study of humidity and ventilator behaviour. Intensive Care Med 2008;34(11):2035-2043.

46. Verbanck S, Paiva M, Schuermans D, Hanon S, Vincken W, Van MA. Relationships between the lung clearance index and conductive and acinar ventilation heterogeneity. J Appl Physiol (1985) 2012; 112(5):782-790.

47. Verbanck S, King GG, Zhou W, Miller A, Thamrin C, Schuermans $\mathrm{D}$, et al. The quantitative link of lung clearance index to bronchial segments affected by bronchiectasis. Thorax 2018;73(1):82-84.

48. Reychler G, Keyeux A, Cremers C, Veriter C, Rodenstein DO, Liistro G. Comparison of lung deposition in two types of nebulization: intrapulmonary percussive ventilation vs jet nebulization. Chest 2004; 125(2):502-508.

49. Reychler G, Wallemacq P, Rodenstein DO, Cumps J, Leal T, Liistro G. Comparison of lung deposition of amikacin by intrapulmonary percussive ventilation and jet nebulization by urinary monitoring. $\mathbf{J}$ Aerosol Med 2006;19(2):199-207. 